\title{
EFFECT OF SHOCKWAVE THERAPY VERSUS LOW- INTENSITY PULSED ULTRASOUND ON BONE HEALING OF MANDIBULAR FRACTURES; (A RANDOMIZED CLINICAL TRIAL)
}

\author{
Eman A. Ahmed ${ }^{1}{ }_{B D s}$, Mostafa M. El Dibany $2 P h D$, Lydia N. Melek $3 P h D$, \\ Hoda M. Abdel Naby $4 P h D$,
}

\begin{abstract}
INTRODUCTION: Low- intensity Pulsed Ultra Sound (LIPUS) and Extra Corporeal Shock Wave Therapy (ESWT), are the different forms of acoustic mechanical waves that might promote bone healing by stimulating bone growth in long or other bones.

OBJECTIVES: To evaluate the effect of adjuvant therapy with LIPUS and ESWT in healing fresh mandibular fractures patients.

MATERIAL AND METHODS: Twenty one patients (12 males, 9 females), aged from 20 to 40 years, had fresh mandibular fractures to be treated with closed reduction and maxillary mandibular fixation (MMF), prospectively. Patients enrolled into this study with ethical approval and informed consent. Those patients were randomly assigned in three treatment groups. Group I received a single treatment with 4000 impulses of focused ESWT, group II received 18 sessions of LIPUS for 20 Min each ; while group III , received neither of those treatments, represented the control group -- Clinical assessment together with radiographic follow-up using Cone Beam Computed Tomography (CBCT) at 1.5 and 3 months were done for all patients postoperatively.

RESULTS: There was statistically significant difference in bone density between three groups in different times after six weeks and after three months as bone density higher in group I than in groups II, III.

CONCLUSIONS: Focused ESWT appeared to be more effective than LIPUS or nothing as an adjuvant treatment of adults with fresh mandibular fractures.

KEYWORDS: Low intensity pulsed ultrasound, extracorporeal shock wave therapy, mandibular fracture.

RUNNING TITLE: ESWT and LIPUS effects on mandibular fracture healing.
\end{abstract}

1 Dentist at Ministry of health, Alexandria, Egypt.

2Professor of Oral and Maxillofacial Surgery, Conservative Dentistry, Faculty of Dentistry, Alexandria University, Alexandria, Egypt.

3Lecturer of Oral and Maxillofacial Surgery, Faculty of Dentistry, Alexandria University, Alexandria, Egypt.

4Lecturer of Physical Medicine Rheumatology and Rehabilitation, Faculty of Medicine, Alexandria University, Alexandria, Egypt.

*Corresponding author:

E-mail: dremanabdelghany@yahoo.com

\section{INTRODUCTION}

Mandibular fractures are the most common fractures found among craniofacial injuries. The pattern and etiology of these injuries varies from one country to another depending on socioeconomic, cultural, and environmental factors. Road traffic accident is the most common etiological factor followed by violence and direct trauma. (1)

The Current methods of management include combinations of soft diet, intermaxillary fixation, open reduction internal fixation, and, rarely, external fixation. Decision-making depends on the age of the patient, type of fracture, medical status and associated injuries. (2)

The main goal of fracture management is the restoration of form and function. In addition, preventing complications like infection, malunion and soft tissue break down. (3)
Reduction of the fracture can be achieved either with an open or closed technique. Closed reduction is indicated in non-displaced favorable fractures, edentulous mandibular fractures, and mandibular fractures in children with developing dentition, as well as coronoid process \& condylar fractures. (4)

Closed reduction with indirect fixation is done by application of maxillomandibular fixation (MMF) for 6 weeks through arch bars, eyelet wiring, Ivy loop, continuous loop or gunning splints. Disadvantages of MMF include inability to directly visualize the reduced fracture, need to keep the patient on a soft diet, and difficulties with speech and respiration.(5) 
Fracture healing is a complex process typically characterized by four overlapping stages: the initial inflammatory response, soft callus formation, hard callus formation, and bone remodeling. (6)

Fracture healing may be modified by several factors such as hormones, vitamins, minerals, local vascularity and microcirculation, weight bearing, protein diet, ultrasound, and electrical stimuli.(7)

The idea of speeding or enhancing mandibular fracture healing to minimize symptoms and inconvenience for the patient is appealing. Bone stimulators such as low intensity pulsed ultrasound (LIPUS) and extracorporeal shock wave therapy (ESWT) might promote bone healing by stimulating bone growth (osteogenesis) in long or other bones through mechanotransduction; physical forces across a cell membrane leading to chemical changes within the cell. The mechanically-induced structural deformation of mechanoreceptors triggers a cascade of biological reactions that assist in healing of fractures. (8)

Low-intensity pulsed ultrasound (LIPUS) is a safe, effective and noninvasive high frequency acoustic therapy used to accelerate the bone healing process. (9)

Distinct physical parameters of LIPUS act on some biological cellular reactions involved in each phase of the healing process such as inflammatory reaction, angiogenesis, chondrogenesis, intra membranous ossification, endochondral ossification, and bone remodeling, so it accelerates bone healing process.(10) The US Food and Drug Administration (FDA) approved the use of LIPUS for accelerating healing of fresh fractures in 1994. There is evidence shows that LIPUS treatment reduces time of fracture healing and improves quality of life. (11)

Currently, many surgeons use LIPUS as part of their management of fractures. However, LIPUS treatment is more suitable for fractures with conservative treatment rather than those with operative treatment as it is a highenergy wave generating deep heat energy that may further aggravate the thermal osteonecrosis caused by bone drilling during the operation. Also, it should not be expected as a method to reduce the incident rate of delayed union and nonunion. (12)

ESWT described as pulsed fast sound waves with a high pressure amplitude and a very short rise time generated by electrohydraulic, electromagnetic, or piezoelectric methods and propagate through tissues. These shock waves stimulate biological effects in living tissue such as antiinflammatory effect, tissue regeneration assist, promote healing, angiogenesis, and bone remodeling .Thus by mechanotransduction and release of energy, in addition to compression and tension of cells all will increase the blood supply to injured area, forming free radicals and oxygen radicals, which lead to the production of a number of different growth factors.(13) Thus promoting osteogenesis, regeneration of fibrocartilage zone, and bone remodeling, hence accelerate fracture healing.(8) ESWT has been used in the treatment of union and nonunion. An increased cortical volume and higher trabecular connectivity was observed after stimulation with ESWT and that leading to improvement of biomechanics of the bone. (13)

Focused shockwaves have an effect on a precisely defined small area, penetrate more deeply and carry more energy to the tissues than radial shockwaves which are more widespread. Focused ESWT increases bone mass density, bone mass concentration in human, improve blood perfusion and thus metabolism of the surrounding soft tissues in addition to its analgesic effect.(14) ESWT has been proven that it is effective as surgical procedures, but more economical and free from side effects. So it should be considered gradually as a first-choice treatment for appropriate non-union fractures. (8)

As the morbidity and socioeconomic costs of fractures are considerable and the management of mandibular fractures may be tricky to achieve an aesthetically practical progress, so the length of time to healing is an important factor in determining a person's recovery after a fracture. It is known that ESWT and LIPUS have a therapeutic role in reducing the time to union after fracture as part of the treatment of acute fractures in adults.(15)

So the aim of this study is to compare between the effect of extracorporeal shockwave Therapy (ESWT) and lowintensity pulsed ultrasound (LIPUS) on bone healing of non-displaced fresh mandibular fractures.

The null hypothesis of this study is that there is no significant difference in mandibular fracture healing in the clinical and radiographic parameters after application of Low-Intensity Pulsed Ultrasound (LIPUS) or Extracorporeal Shock Wave Therapy (ESWT).

\section{MATERIALS AND METHODS}

Study design:

The study was a randomized controlled clinical trial performed on twenty-one patients with fresh favorable mandibular fractures. Both genders were selected of age range from 20 to 40 years from the outpatient Clinic of Oral and Maxillofacial Surgery Department, Faculty of Dentistry, Alexandria University, Egypt, and from the Emergency Ward of the Alexandria Main University Hospital. Faculty of Medicine , University Of Alexandria, Egypt.

The study was performed after gaining the approval of the Research Ethics Committee, Faculty of Dentistry , Alexandria University.

A full Informed consent was taken from all patients after explaining all the procedures to the patient including all benefits and side effects simply and easily also, the patients had the right to withdrawal at any time.

Materials used

- Wire and arch bar used in inter-maxillary fixation (Erich arch bar or eyelet wiring 24 gauge stainless steel wire) (Figure 1.A ,B ).

- Extracorporeal Shockwave therapy device (The orthowave 180c , MTS Europe Gmbh, Robet-Bosch-Str. 18, D-78467 Konstanz, Germany, Serial No. 180c00089, Type/model Orthowave 180c, Nominal Supply Voltage 
230V AC, Max. Power Consumption 500VA, Power Supply Frequency 50Hz, Class I, according to IEC 601-1) (Figure1,C).

- Low- intensity pulsed ultrasound Device(Sonopuls 692 Enraf-Nonius B.V, P.O. Box 12080, Vareseweg 127, 3004 GB Rotterdam, The Netherlands, REF. 1600.945, SN. 03.877, 100240V /50-60Hz/130VA, June 2011) (Figure1,D).
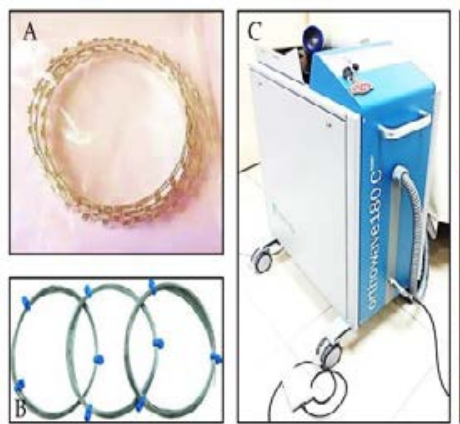

Figure (1): (A) Erich arch bar.

(B) 24 gauge stainless steel wire.

(C) Orthowave $180 \mathrm{c} \circledR$.

(D) Low- intensity pulsed ultrasound Device

\section{Methods}

The sample size was determined by the department's professor's suggestion during the department council meeting and discussion of the protocol. Seven patients were identified in each group as a minimum due to the high cost of physiotherapy sessions.

The trial was registered at clinical trial. gov. (NCT04518956)

All fractures were single non-displaced fresh mandibular fractures treated with closed reduction and maxillary mandibular fixation (MMF)

Inclusion criteria

a) A through history was recorded in a standardized format. Patients were from both genders, males and females, patients' age ranges from 20 to 40 years, all patients were free of any systemic or bone disease. Patients presenting with infection, burn and scar in the skin of relevant working area or with any contraindication to ESWT, LIPUS or CBCT were excluded.

b) They should had single non-displaced fresh fracture ( recent fracture not neglected nor infected and not more than one week old) with no communication to the external environment.

c) The sites of the mandibular fractures accepted were symphyseal, Parasymphyseal, body , angle and ramus) indicated for treated with closed reduction and maxillary mandibular fixation (MMF).

Exclusion criteria

a) Patients with bone diseases affecting the bone healing process.

b) Patients presenting with infection, burn and scar in the skin of relevant working area.

c) Patients not willing to return for follow up.

Pre-Surgical Assessment

A-History of patient
Personal history : data was collected and recorded in full details including name, age, gender, occupation, telephone number have been obtained and address. Past medical history of any systemic diseases as well as drug history and dental history.

Chief complaint, All details about the trauma were recorded including cause, time, date, place and type of assault, and specification of the injury such as (the type of the object and the direction from which the contact was made), detection of any sign of nerve injury anaesthesia or parathesia.

B-Clinical examination

It was performed both extra orally and intraorally

Extra oral examination

- Inspection to detect any swelling, ecchymosis, deformity, deviation during mouth opening and closure. Jaw deviation during function and soft tissue laceration.

- Palpation of the mandible was carried out by fingers of both hands while the thumbs were placed on the lateral aspects, starting at the midline fingers passed posteriorly to assess any step deformity, alteration in the bone contour, tenderness, condylar movements during opening and closing, dislocation, altered lip sensation and bony crepitus.

Intraoral examination

- Inspection for bleeding, hematoma, ecchymosis, occlusal disharmony or malocclusion, broken teeth, alignment of teeth, integrity of teeth, shift in the mid line in opening and closing of the mouth and mandibular movement (opening, lateral and protrusive movement).

- Palpation of buccal and lingual sulci for the presence of tenderness or alteration in the contour. Bimanual manipulation of the mandible on either side of the suspected fracture was made for the detection of mobility and tenderness. Mental nerve innervation was compared with the opposite side to conclude presence of anaesthesia or paraesthesia.

Radiographic examination

Standard Preoperative Orthopantomogram (OPG) of the mandible were done for all patients at the time of entrance to evaluate the condition of the mandibular fracture, teeth in the line of fracture and degree of displacement

Preoperative preparation

The oral cavity should be prepared before starting of the treatment by performing oral hygiene measures, scaling, rest treatment for any carious tooth, and suturing of any soft tissue laceration under local anaethesia, Intraoral wound was closed using Vicryl suture material $(90 \%$ glycolide and 10\% L-lactide: Ethicon part of Johnson \& Johnson Int, US.) while extra oral incision was managed in layers using Vicryl sutures for the deep layers and Prolene sutures for the skin (polypropylene: Ethicon part of Johnson \& Johnson Int, US.) Intravenous cefotaxime 1 $\mathrm{gm} / 12$ hours for the first day followed by (Amoxicillin 875mg + clavulanic acid 125mg: GlaxoSmithKline, UK) $1 \mathrm{gm}$ twice daily for the next 5 days, Metronidazole (500mg: GlaxoSmithKline, UK.) every eight hours for 5 days), (Diclofenac potassium 50mg: Novartis- 
Switzerland). Every eight hours for 5 days. All patients were instructed to rinse their mouth using (chlorhexidine $125 \mathrm{mg} / 100 \mathrm{ml}$, concentration 0.125\%: Arabic drug company, ADCO).

Operative phase

All patients were operated under local anaesthesia2\% lidocaine (1:100000 epinephrine) (Amoun Pharmaceutical - Egypt), fmanagement of teeth in the fracture line was done by preservation according to the preoperative assessment.

- Closed reduction and fixation is done by application of maxillary mandibular fixation (MMF) which is indicated for non-displaced favourable fractures, Ivy loops or Erich arch bars were fixed to maxillary and mandibular present teeth using 24 gauge wires.

Oral hygiene measures were stressed, including daily brushing of the teeth and arch bars and rinsing by using antiseptic mouth wash, the patient was maintained on a soft diet, adequate medication for good pain relief and antibiotics for good infection control.

For group (I) (study group), Extracorporeal shockwave therapy was applied in which seven patient received a single session of high energy electrohydraulic shock wave therapy the day after closed reduction (MMF) (2000-4000 impulses; $0.35 \mathrm{~mJ} / \mathrm{mm} 2$ ) energy flux density per impulse in 20 minute, with frequency range from 1 to 5 pulse per second under regional anaesthesia.

For group (II) (study group) of low intensity pulsed ultrasound seven patients underwent the day after closed reduction (MMF) eighteen sessions of 20 minutes through which high frequency sound waves and vibration with frequency of $1 \mathrm{MHz}$, intensity $1.50 \mathrm{~W} / \mathrm{Cm} 2$ continuous were applied on the surface of the skin at the area of fracture line, repeated three times weekly for six weeks.

For group (III) (control group) seven patients in these group their fractures were reduced by maxillary mandibular fixation only.

\section{Postoperative phase}

-Warm fomentation, antibiotics, anti-inflammatory and analgesic drugs (Diclofenac Potassium orally $50 \mathrm{mg}$ three time daily postoperatively for a period of 5 days. Patients were instructed for careful oral hygiene measures was maintained by $0.2 \%$ chlorhexidine oral rinses., stop smoking and avoid biting on any hard food and rest. In addition soft, fully liquid, high protein, high calorie diet was given for all patients for 4 weeks postoperatively. A minimum post-operative follow-up period of 3 months was required.

Delayed postoperative care

Clinical and radiographic follow-up was performed for all patients for three months postoperatively.

Clinical follow-up

Clinical follow-up was carried out after 24 -hours, one week, four weeks, six weeks and twelve weeks postoperatively. Clinical assessment was achieved through Postoperative Pain which was determined through a 10point Visual Analogue Scale (VAS). The patients were asked to rate their postoperative pain and discomfort from 0 to $10(0-1=$ None, $2-4=$ Mild, 5-7= Moderate, $8-10=$ Severe $)$.

Radiographic evaluation Cone beam computed tomography (CBCT) was acquired with J.Morita R100 cone beam 3D imaging system (Morita 3DX; J Morita Mfg corp., Kyoto, Japan). The scan was done with field of view (FOV) W 100mm X H $80 \mathrm{~mm}$. The volume was reconstructed with $0.250 \mathrm{~mm}$ isometric voxel size. The tube voltage was $90 \mathrm{kVp}$ and $8 \mathrm{~mA}$, Exposure time was 20 seconds. The image was analyzed using OnDemand3D ${ }^{\mathrm{TM}}$ software (Cybermed Inc.) CBCT analyzing software. The mean bone density at the site of fracture was calculated by measuring the bone density at 6 points distributed inside the fracture line then taking their mean values to determine the mean bone density at 6 weeks and 3 months postoperatively.

All the obtained data was statistically analysed and presented in tables, graphs and charts using the IBM Statistical Package for Social Science (SPSS)

Kruskal Wallis test, Pairwise comparison bet. Each 2 groups was done using Post Hoc Test (Dunn's for multiple comparisons test) was applied to determine any significant differences in pain, occurrence of complications, mean bone density between the patients at different follow up periods.

\section{RESULTS}

The demographical data of this study; including the gender and age of the participants were as follows: 12 males and 9 female patients participated in this study. The ages of the participants ranged from 21.0 to 38.0 with an average of $28.52 \pm 5.50$,

Road traffic accident is the most common etiological factors of mandibular fractures in $71.4 \%$ of patients, interpersonal violence in $23.8 \%$ and fall in $4.8 \%$.

The anatomic positions of mandibular fractures were parasymphysis in $38.1 \%$, the body in $47.6 \%$ and ramus in $14.3 \%$ of patients. No complications were observed except for slight gingival inflammation and bleeding after MMF in immediate postoperative period.

Regarding pain intensity, it was observed during the first 24 hours after treatment that the mean value of pain score using VAS in group I was $8.14 \pm 0.69$, group II was 7.71 \pm 0.76 while in the control group III was $7.86 \pm 0.69$.But this difference was not statistically significant as $p$ value $=0.500(\mathrm{p} \leq 0.05)$.

One week later, a statistically significant decrease in pain was observed in group I $(2.86 \pm 1.07)$, group II $(4.86 \pm 0.90)$ as well as in group III $(6.0 \pm 0.58) \mathrm{p}$ value $=$ 0.001 ( $\mathrm{p} \leq 0.05)$. However, the decrease of pain in group I was statistically significant in comparison to either group II and III (p1=0.032*, p2<0.001*respectively) but not on comparing groups II and III (p3=0.094).

(Figure 2, Table 1) 
Table (1): Distribution of the studied cases at two periods according to pain $(n=21)$

\begin{tabular}{|l|c|c|c|c||}
\hline \multicolumn{1}{|c|}{ Pain } & 1st time & 2nd time & $\mathrm{Z}$ & $\mathrm{p}$ \\
\hline Shock wave & & & & \\
Min. -Max. & $7.0-9.0$ & $2.0-4.0$ & & \\
Mean \pm SD. & $8.14 \pm 0.69$ & $2.86 \pm 1.07$ & $2.379^{*}$ & $0.017^{*}$ \\
Median & 8.0 & 2.0 & & \\
\hline Ultrasound & & & & \\
Min. -Max. & $7.0-9.0$ & $4.0-6.0$ & & \\
Mean \pm SD. & $7.71 \pm 0.76$ & $4.86 \pm 0.90$ & $2.392^{*}$ & $0.017^{*}$ \\
Median & 8.0 & 5.0 & & \\
\hline Control & & & & \\
Min. -Max. & $7.0-9.0$ & $5.0-7.0$ & & \\
Mean \pm SD. & $7.86 \pm 0.69$ & $6.0 \pm 0.58$ & $2.414^{*}$ & $0.016^{*}$ \\
Median & 8.0 & 6.0 & & \\
\hline
\end{tabular}

Z: Wilcoxon signed ranks test

$\mathrm{p}$ : $\mathrm{p}$ value for comparing between the studied periods

*: Statistically significant at $\mathrm{p} \leq 0.05$

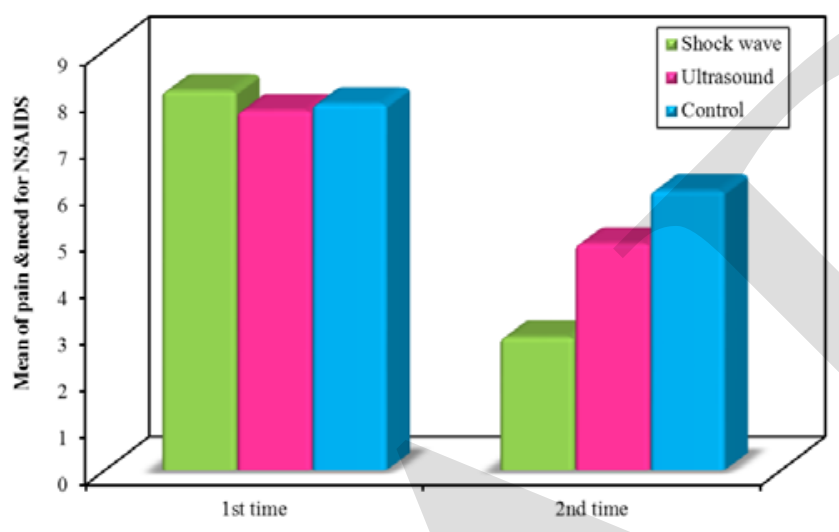

Figure (2): Descriptive analysis of the three studied groups at two times according to pain $(n=21)$.

At one and half months postoperatively, the mean bone density in group I was $1145.7 \pm 166.5$ voxel value (VV), group II was $535.9 \pm 190.5 \mathrm{VV}$ while in control group III it was $803.05 \pm 197.8 \mathrm{VV}$, the difference between the three groups was found to be statistically significant ( $p$ $=0.001 ; \mathrm{p} \leq 0.05$ ). Significant higher mean bone densities was found in group I compared to group II or III $(\mathrm{p} 1<0$. 001*, p2 $=0.025 *$ respectively). At three months postoperatively, the mean bone density was $1472.3 \pm 24.09$ $\mathrm{VV}$ in group I, $612.5 \pm 92.4 \mathrm{VV}$ in group II and 960.0 \pm 198.0 VV in the control group III, again the difference between the three groups was found to be statistically significant $(p=0.001 ; p \leq 0.05)$. (Figure 3 , Table 2$)$

The percentage change in the bone density after one and half to 3 months' postoperative measurements was not statistically significant comparing the three groups to each other. This means that group I with the use of ESWT attained a statistically significant higher bone densities at one and half months post-operatively than the other two groups, which maintained the same effect at 3 months period. (Figure 4, 5, 6, Table 3)
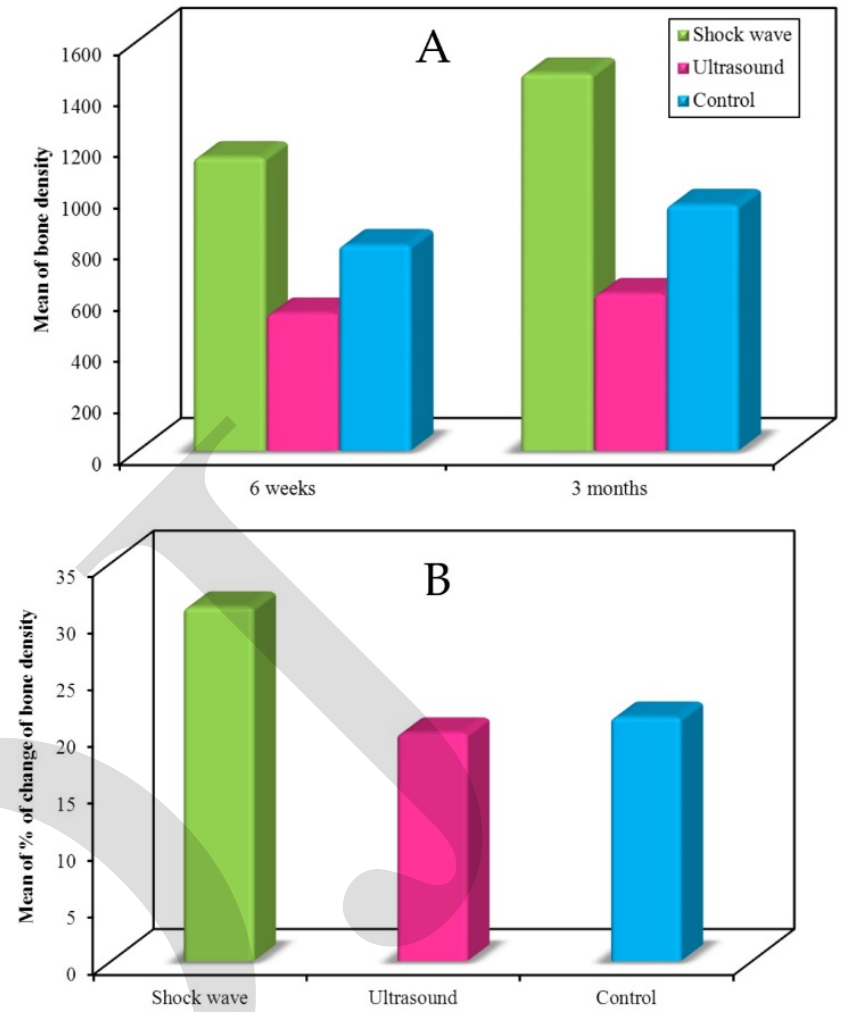

Figure (3): (A) Descriptive analysis of the three studied groups at two different periods of time according to bone density $(n=21)$. (B) Distribution of the studied groups at two different times according to \% of change of bone density ( $\mathrm{n}=21)$

Table (2): Distribution of the studied groups at two times according to bone density $(n=21)$

\begin{tabular}{|c|c|c|c|c|}
\hline $\begin{array}{c}\text { Bone } \\
\text { density }\end{array}$ & $\begin{array}{l}6 \text { weeks post } \\
\text { operatively }\end{array}$ & $\begin{array}{c}3 \text { months post } \\
\text { operatively }\end{array}$ & Z & $\mathrm{p}$ \\
\hline $\begin{array}{l}\text { Shock wave } \\
\text { Min. -Max. } \\
\text { Mean } \pm \text { SD. } \\
\text { Median }\end{array}$ & $\begin{array}{c}855.9-1334.5 \\
1145.7 \pm 166.5 \\
1150.17\end{array}$ & $\begin{array}{c}1430-1495 \\
1472.3 \pm 24.09 \\
1480.0\end{array}$ & 2.366* & $0.018^{*}$ \\
\hline $\begin{array}{l}\text { Ultrasound } \\
\text { Min. -Max. } \\
\text { Mean } \pm \text { SD. } \\
\text { Median }\end{array}$ & $\begin{array}{c}364.3-930.8 \\
535.9 \pm 190.5 \\
494.3\end{array}$ & $\begin{array}{c}505.9-760.7 \\
612.5 \pm 92.4 \\
622.0\end{array}$ & 1.352 & 0.176 \\
\hline $\begin{array}{l}\text { Control } \\
\text { Min. -Max. } \\
\text { Mean } \pm \text { SD. } \\
\text { Median }\end{array}$ & $\begin{array}{c}376.2-957.28 \\
803.05 \pm 197.8 \\
833.83\end{array}$ & $\begin{array}{c}526.2-1100.6 \\
960.0 \pm 198.0 \\
1020.5\end{array}$ & $2.366^{*}$ & $0.018^{*}$ \\
\hline
\end{tabular}

Z: Wilcoxon signed ranks test

$\mathrm{p}$ : $\mathrm{p}$ value for comparing between the studied periods

*: Statistically significant at $\mathrm{p} \leq 0.05$ 

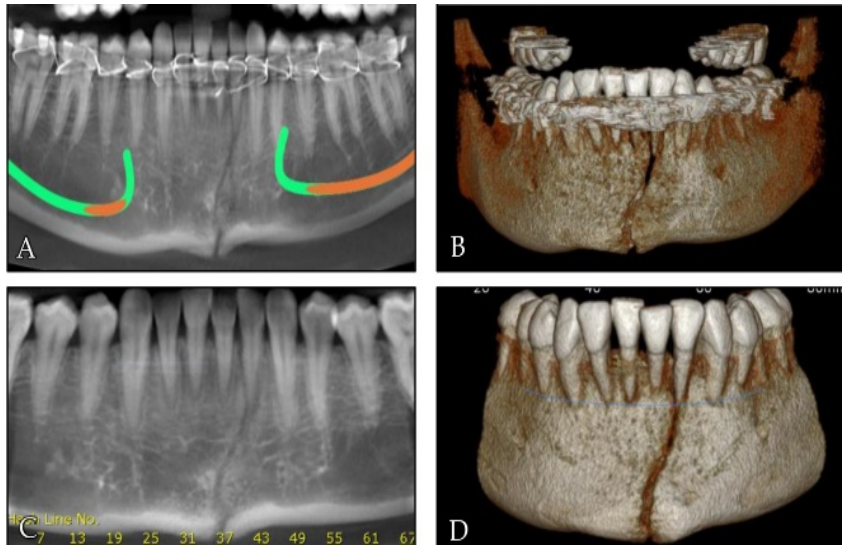

Figure (4): A 31-year-old male patient with left parasymphyseal fracture treated with closed reduction and MMF then received one session of ESWT. a,b panoramic and 3D CBCT sections I.5 months post-operatively showing early signs of fracture healing . c,d panoramic and 3D CBCT sections 3 months post-operatively showing progression of bone healing.
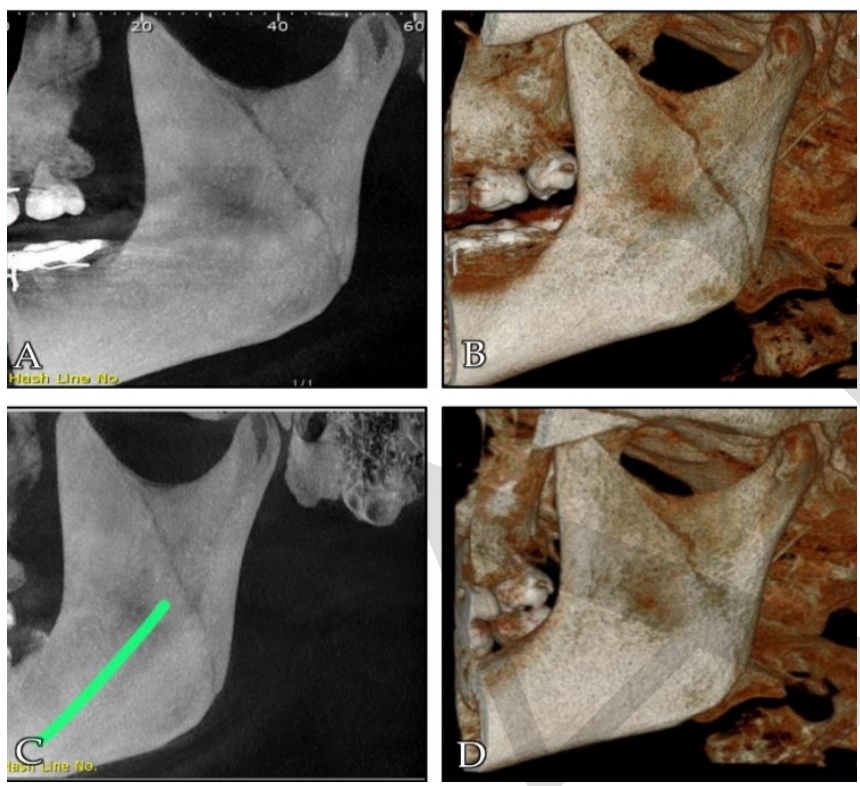

Figure (5): A 27-year-old female patient with left ramus fracture treated with closed reduction and MMF then received 18 sessions of LIPUS. a,b panoramic and 3D CBCT sections I.5 months post-operatively showing early signs of fracture healing, c,d panoramic and 3D CBCT sections 3 months post-operatively showing progression of bone healing

Table (3): Distribution of the studied groups at two different times according to $\%$ of change of bone density $(n=21)$

\begin{tabular}{|c|c|c|c|c|c|}
\hline $\begin{array}{c}\% \text { of change } \\
\text { of bone } \\
\text { density from } \\
6 \text { weeks to } 3 \\
\text { months }\end{array}$ & $\begin{array}{c}\text { Shock wave } \\
(\mathrm{n}=7)\end{array}$ & $\begin{array}{c}\text { Ultrasound } \\
(\mathrm{n}=7)\end{array}$ & $\begin{array}{l}\text { Control } \\
(\mathrm{n}=7)\end{array}$ & $\mathrm{H}$ & $\mathrm{p}$ \\
\hline Min. -Max. & $7.15-71.81$ & $-18.28-40.07$ & $9.76-39.86$ & & \\
\hline Mean \pm SD. & $31.11 \pm 21.22$ & $20.04 \pm 19.88$ & $21.44 \pm 9.92$ & 0.742 & 0.690 \\
\hline Median & 28.68 & 18.52 & 18.09 & & \\
\hline
\end{tabular}

H: H for Kruskal Wallis test

$\mathrm{p}$ : p value for comparing between the studied groups
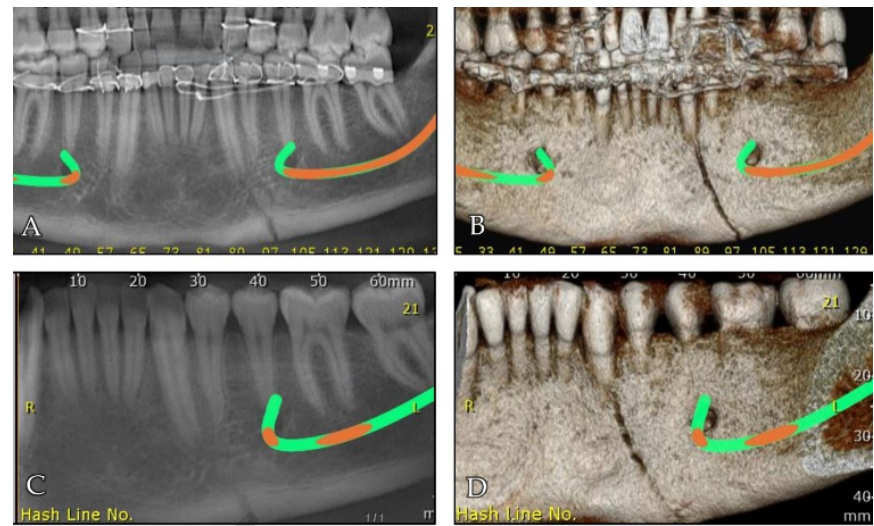

Figure (6): A 35-year-old male patient with left parasymphyseal fracture treated with reduction and maxillary mandibular fixation, a,b panoramic and 3D CBCTsections I.5 months post-operatively showing early signs of fracture healing, c,d panoramic and 3D CBCT sections 3 months post-operatively showing progression of bone healing .

\section{DISCUSSION}

Fracture healing is highly complex process affected by many biological and biomechanical factors. Optimizing the conditions for healing is the basis and the goal of all fracture treatment. Postoperative morbidity stills a major disadvantage following oral and maxillofacial surgery with pain, trismus, swelling and infection that affect patients' quality of life and psychological wellbeing. (16)

Even in conservative management of undisplaced or minimally displaced mandibular fractures by MMF, unfortunately, it requires a prolonged period of immobilization which causes discomfort, weight loss, malnutrition, and most importantly can compromise airway function in case of vomiting. In an attempt to reduce immobilization time so facilitating early return to function with minimal morbidity, there were lots of ways and studies about how to accelerate bone healing to ensure the patients rapid recovery which includes return to work, recreation and family life. Enhancement of biomechanical environment of skeletal repair can be done by physical stimulation therapies also known as bone growth stimulators. Those modalities are appealing because they are almost safe and less invasive. The two current major categories of physical stimulation therapies used for fracture healing include low intensity pulsed ultrasound, and extracorporeal shock wave therapy. (17)

So in our study we investigated the effect of both devices on the healing of fresh mandibular fractures, the extracorporeal shock wave therapy for group I (study group), and low intensity pulsed ultrasound for group II (study group) and no application of physical stimulation therapy for group III (control group).

Twenty-one Egyptian adults aged 20-40 years old with fresh mandibular fractures were included in this study. All patients were free of any systemic or bone disease. So excluding any patient- related factors affecting fracture 
healing. In addition, patients presenting with infection, burn and scar in the skin of relevant working area or with any contraindication to ESWT, LIPUS or CBCT were excluded. Furthermore, all fractures were single nondisplaced fresh mandibular fractures treated with closed reduction and MMF using Erich arch bar or eyelet wiring fixed using 24 gauge wires.

Twelve patients were males (57.1\%) and nine were females. Male affection was more which is consistent with most of the studies. This is in accordance with other studies showing a male predilection in maxillofacial fracture. (18-20)

Road traffic accident was the most common etiologic factor $(71.4 \%)$, then interpersonal violence (23.8\%) and fall (4.8\%). Young adults represent a large mass in our country who actively participate in outdoor activities without much caring about safety rules such as seat belts in cars and wearing crash helmets when riding motorbikes. Over using mobile phones while driving with noncompliance with traffic laws.Next come inter personal violence and falls as a cause of maxillofacial injuries in which sport related injuries seem to be increasingly implicated in the etiology of maxillofacial trauma.(20)

In the present study the most frequent anatomic locations of mandibular fractures were in the body of the mandible (47.6\%), followed by parasymphyseal region (38.1\%) and ramus in $14.3 \%$. This is mostly regarding to the fact that lower canines having longer roots than adjacent teeth, the fact that makes the bone in this area weaker and more liable to fracture. These results are incompatible with Sakr et al., who found in 2006 that the angle was the most common site.(20) and with Melek and Sharara, who found in 2016 that parasymphyseal region is the most commonly affected site.(21) Still, no complications were observed except for slight gingival inflammation and bleeding after MMF in the immediate postoperative period.

The pre-operative evaluation was based on clinical, extraoral, intra-oral examination and standard Orthopantomogram to assess the site of fracture and exclude other associated injuries. In all patients, standard principles of surgical preparation, reduction, fixation and suturing were followed. And oral hygiene was maintained by $0.2 \%$ chlorhexidine oral rinses. Warm fomentation, antibiotics, anti-inflammatory and analgesic drugs were administered for 5-7 days following surgery. Patients were instructed for careful oral hygiene measures, stop smoking and avoid biting on any hard food and rest. In addition soft, fully liquid, high protein, high calorie diet was given for all patients for 4 weeks postoperatively. A minimum post-operative follow-up period of 3 months was required. In group I (seven patients) the fractures were closely reduced, treated with maxillary mandibular fixation (MMF), and followed by receiving a single session of high energy electrohydraulic focused shock wave therapy (4000 impulses; $0.35 \mathrm{~mJ} / \mathrm{mm} 2$ ) energy flux density per impulse and frequency range from 4 to $6 \mathrm{~Hz}$ under regional anaesthesia. It had been postulated that the extracorporeal shockwaves caused micro trauma or micro fracture and induced neovascularization through hematoma formation which would increase osteoblast or fibroblast activity. Focused shockwave affects small precisely defined area carrying more energy which invades deeply in tissues to increase local blood flow and stimulates inflammatory response to promote tissue healing accordingly.(14) The biomechanical pathway promoted by dynamization and local strain or deformation enhancing micro-movement at the fracture site resulting in stimulation of osteogenesis.(22)

Modern research efforts in orthopaedics are oriented into bone callus enhancement by surgical, pharmacological, cellular, and biophysical strategies in order to improve fracture healing process. (23)

In previous studies, the authors concluded that extracorporeal shock wave therapy may stimulate the healing process as it produces physiological effects and biological healing processes, bone remodeling, antiinflammation and increases bone mass concentration, in delayed unions or nonunion. (15)

Several studies have strongly backed the evidence that ESWT has a potential to induce bone regeneration. It was concluded that ESWT combined with intermaxillary fixation can be an effective therapy for accelerated fracture healing and even for the reduction of complications associated with fracture healing.(8)

In group II (seven patients) with mandibular fracture which was closely reduced and fixed with MMF, and then underwent eighteen sessions for twenty minutes of low intensity pulsed ultrasound with frequency of $1 \mathrm{MHz}$, intensity $1.50 \mathrm{~W} / \mathrm{cm} 2$ applied externally over the area of fracture line, starting from the day after closed reduction and repeated three times weekly for six weeks with considering analgesic if required on the day of the session. LIPUS was found to enhance fracture healing by stimulating earlier synthesis of extracellular matrix protein, the aggrecan in cartilage, possibly altering chondrocyte maturation through endochondral bone formation pathway. Other clinical trials; done on humans, have shown that LIPUS has a positive effect on bone healing and the reduction of fracture healing time, mainly in cases with long bone fractures.(23)

LIPUS treatment is more suitable for fractures with conservative treatment rather than those with operative treatment as it is a high-energy wave generating deep heat energy that may further aggravate the thermal osteonecrosis caused by bone drilling during the operation. Also, it should not be expected as a method to reduce the incident rate of delayed union and nonunion. $(24,25)$

The third group III (seven patients) their mandibular fractures were closely reduced and fixed with MMF, by using Erich arch bar and 24 gauge stainless steel wire without any physical intervention.

Regarding the postoperative pain assessed by visual analogue scale (VAS). There was no significant difference between the three groups at the first 24 hours

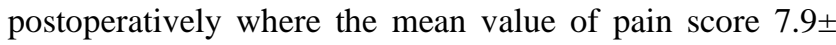


0.7 in the three groups, and was treated by using diclofenac potassium orally $50 \mathrm{mg}$ three times daily for five days. After one week, pain score was measured again by using VAS and the mean value of pain score in group I (ESWT group) which was subjected to shock wave session became $2.86 \pm 1.07$. For group II (LIPUS group) the mean value of pain score became $4.86 \pm 0.90$ after 3 sessions of low intensity pulsed ultrasound, where in group III (control group) the mean value of pain score became $6.0 \pm 0.58$.This difference was statistically significant where the pain decreased markedly after one week in case of group I. This may be explained by the fact that extracorporeal shock wave improves the blood circulation and has a proven analgesic effect. $(8,15)$

In this study, radiographic follow up was performed with CBCT radiograph to measure the bone density. This agreed with the studies of Angelopoulos et al. 2012 (26), and Parsa et al. 2012 (27) which demonstrate a strong correlation between gray- scale values of CBCT images and bone densities. This has implications for potential quantitative radiological approaches to determine bone density from CBCT images. The mean bone density of group I was higher than groups II and III, but the percentage change of the bone density was not statistically significant comparing the three groups.

Our results show that the use of the extracorporeal shock waves as an adjuvant in the treatment of fractures of the human lower jaw is better as its applicator produces shock waves which are single high amplitude sound waves generated by electrohydraulic generator that provides a deeper penetration (up to $5 \mathrm{~cm}$ ) than that of conventional ultrasound therapy. Shock waves differ from regular sound waves in that when compression takes place, there is sudden change in stress and density. Also, they travel faster than sound, and their speed increases as the amplitude (pressure) is raised. And as the intensity of a shock wave decreases faster than does of a sound wave, wavelets at high pressure lead to deformation of the wave so that the wave crest assumes a sawtooth appearance, which is different from the sinusoidal appearance of a regular sound wave. Furthermore, shock waves differ from ultrasound waves since the former is uniphasic with high peak pressure (in the order of a hundred $\mathrm{MPa}$ ), and the latter is biphasic with very low peak pressure (in the order of a hundredth of MPa). (24)

Shock wave has shown to be a less time consuming (20 minutes for one single session) than the low intensity pulsed ultrasound (20 minutes per day, three sessions weekly for six weeks). $(28,29)$

In conclusion of ESWT application on the fracture site of mandibular fracture may be useful in acceleration of the fracture healing and our study could be the setting stone for further studies and researches as to the best of our knowledge, it is the first time for using shock wave therapy in the management of jaw bone fractures. So we reject the null hypothesis of this study.

This study has some limitations. The sample size was small and included a homogenous population of patients. We didn't include children or elderly whom may benefit a lot from accelerating healing and analgesic effect of ESWT. Also, patients with biological factors known to affect bone healing like anemia, malnutrition, smoking, diabetes mellitus, vasculopathies and others either patient-related or fracture-related were not included. These issues may be the target of future large-sized clinical trials.

\section{CONCLUSIONS}

Within the limitation of this study, the following could be concluded:

Extracorporeal shock wave therapy is safe and effective therapy for fracture healing as it can increase the bone mass density, advance blood perfusion and metabolism of the surrounding soft tissue, In addition to play an important role in pain-relieving effect. So it may be helpful in acceleration of fracture mandible.

This study is the first time of using shock wave therapy in the treatment of jaw bone fractures and the promising results will encourage the implementation of more studies to confirm the value of this treatment modality.

\section{Conflict of interest}

The authors declare that they have no conflicts of interest.

\section{REFERENCES}

1. Arabion, H. R., Tabrizi, R., Aliabadi, E., Gholami, M., \& Zarei, K. A retrospective analysis of maxillofacial trauma in shiraz, iran: a 6-year-study of 768 patients (2004-2010). Journal of Dentistry 2015:15;15.

2. Eusterman, V. D. Maxillofacial Trauma. In Brennan, J. A., Richard, G. H., Connor, M. P., Donald, P. J., Eusterman, V. D., Hayes, D. K., Kellman, R. M. (editors). Cumming's Otolaryngology: Head and Neck Surgery. Ch5, USA; 2012:100-39. The American Academy of Otolaryngology.

3. Stacey DH, Doyle JF, Mount DL, Snyder MC, Gutowski KA. Management of mandible fractures. Plast Reconstr Surg 2006 Mar; 117(3):48e-60e.

4. Ochs MW, Tucker MR. Management of Facial Fracture. In: Larry J Peterson,

5. Contemporary Oral and Maxillofacial Surgery, 4th edition, Ch24, Mosby, USA, 2005, 537.

6. Anil PP, Alan SH. Mandibular fractures. In: Thaller SR, Mcdonlad WS. Facial trauma. Ch16, Marcel Dekker Inc, New York, 2004, 383.

7. Schindeler A, Mc Donald MM, Bokko P, Little DG,. Bone remodeling during fracture repair: The cellular picture. Semin Cell DevBiol 2008; 19:459-66.

8. Siska, P. A., Gruen, G. S., \& Pape, H. C. External adjuncts to enhance fracture healing: What is the role of ultrasound?. Injury 2008; 39, 1095-105.

9. Cheng, J. H., \& Wang, C. J. Biological mechanism of shockwave in bone. International Journal of Surgery 2015;24, 143-6.

10. Watanabe Y1, Matsushita T, Bhandari M, Zdero R, Schemitsch EH. Ultrasound for fracture healing: current evidence. J Orthop Trauma. 2010 Mar;24 Suppl 1:S56-61. 
11. Harrison A, Lin S, Pounder N, Mikuni-Takagaki Y. Mode \& mechanism of low intensity pulsed ultrasound (LIPUS) in fracture repair. Ultrasonics 2016 Aug;70:45-52.

12. Rutten S, van den Bekerom MP, Sierevelt IN, Nolte PA. Enhancement of Bone-Healing by Low-Intensity Pulsed Ultrasound: A Systematic Review. JBJS Rev. 2016 Mar 29;4(3).

13. Lou S, Lv H, Li Z, Tang P, Wang Y. Effect of lowintensity pulsed ultrasound on distraction osteogenesis: a systematic review and meta-analysis of randomized controlled trials. J Orthop Surg Res. 2018 Aug 17;13(1):205.

14. Van der Jagt, O. P., Waarsing, J. H., Kops, N., Schaden, W., Jahr, H., Verhaar, J. A., \& Weinans, H.. Unfocused extracorporeal shock waves induce anabolic effects in osteoporotic rats. Journal of Orthopaedic Research 2013; 31, 768-75.

15. Schaden, W., Mittermayr, R., Haffner, N., Smolen, D., Gerdesmeyer, L., \& Wang, C. J. Extracorporeal shockwave therapy (ESWT)-First choice treatment of fracture nonunions?. International Journal of Surgery 2015; 24, 179-83.

16. Griffin, X. L., Parsons, N., Costa, M. L., \& Metcalfe, D. Ultrasound and shockwave therapy for acute fractures in adults. Cochrane Database of Systematic Reviews 2014, 23, CD008579.

17. Ristow, O., Pautke, C., Kehl, V., Koerdt, S., Hahnefeld, L., \& Hohlweg-Majert, B. Kinesiologic taping reduces morbidity after oral and maxillofacial surgery: a pooled analysis. Physiotherapy Theory and Practice 2014; 30, 390-8.

18. Kamath, R. A., Bharani, S., Hammannavar, R., Ingle, S. P., \& Shah, A. G. Maxillofacial trauma in central karnataka, India: an outcome of 95 cases in a regional trauma care centre. Craniomaxillofacial trauma \& reconstruction 2012; 5, 197-204.

19. Natu, S. S., Pradhan, H., Gupta, H., Alam, S., Gupta, S., Pradhan, R., ... \& Agarwal, A. An epidemiological study on pattern and incidence of mandibular fractures. Plastic Surgery International; 2012, 834:64.

20.Sakr, K., Farag, I. A., \& Zeitoun, I. M. Review of 509 mandibular fractures treated at the University Hospital, Alexandria, Egypt. British Journal of Oral and Maxillofacial Surgery 2006; 44, 107-11.
21. Melek, L. N., \& Sharara, A. A. Retrospective study of maxillofacial trauma in Alexandria University: Analysis of 177 cases. Tanta Dental Journal 2016: 13, 28-33.

22. Glatt, V, Evans, C, Tetsworth, K. A Concert between Biology and Biomechanics: The Influence of the Mechanical Environment on Bone Healing. Frontiers in Physiology; 2017:1(7).

23. Marongiu G, Dolci A, Verona M, Capone A. The biology and treatment of acute longbones diaphyseal fractures: Overview of the current options for bone healing enhancement.

24. Bone Rep. 2020 Jan;12:100249

25. Warden, S. J., Fuchs, R. K., Kessler, C. K., Avin, K. G., Cardinal, R. E., \& Stewart, R. L. Ultrasound produced by a conventional therapeutic ultrasound unit accelerates fracture repair. Physical Therapy 2006; 86, 1118-27.

26. Angelopoulos, C., Scarfe, W. C., \& Farman, A. G. A comparison of maxillofacial CBCT and medical CT. Atlas of the oral and maxillofacial surgery clinics of North America2012; 20, 1-17.

27.Parsa, A., Ibrahim, N., Hassan, B., Motroni, A., der Stelt, P. V., \& Wismeijer, D. Reliability of voxel gray values in cone beam computed tomography for preoperative implant planning assessment. International Journal of Oral and Maxillofacial Implants 2012; 27, 1438.

28. Zelle, B. A., Gollwitzer, H., Zlowodzki, M., \& Bühren, V. Extracorporeal shock wave therapy: current evidence. Journal of orthopaedic trauma 2010;24, S66-70.

29. Ebrahim, S., Mollon, B., Bance, S., Busse, J. W., \& Bhandari, M. Low-intensity pulsed ultrasonography versus electrical stimulation for fracture healing: a systematic review and network meta-analysis. Canadian Journal of Surgery 2014;57:e105-18. 